\title{
A NEW PROPOSED APPROACH FOR SOLVING THE COMBINED ORDER DESIGN PROBLEM
}

\author{
Rahil, Abo El Hassan, \\ Civil Eng. Dep., Faculty of Eng., Minufiya University, Egypt.
}

\begin{abstract}
The optimization of geodetic networks may be classified into five orders (Kuang, 1996), namely: the zero-order design problem (ZOD), which is the design of the optimal reference system (datum); the first-order design problem (FOD), which aims to optimize the configuration of the network (that is the point locations and the types of observed quantities and its distribution all over the network); the second-order design problem (SOD), which aims to find the optimal weights of the observations; the combined-order design problem (COMD), where both the first-and secondorder design problems have to be optimally solved simultaneously; and finally the third-order design problem (THOD), which is the improvement of an existing design, by densifying or extending the existing networks, by additional points and/or observations (Grafarend and Schaffrin, 1979).

The COMD problem is classified, according to the present study, into three types: trial and error approach, analytical approach and semi-analytical approach (Doma (2004)). The main objective of this paper is to introduce a new suggested approach for solving the combined order design (COMD) problem. Also, the discussion for solving the COMD problem is given. Three computer programs for the computation of the criterion matrix, solving the second-order design (SOD) problem and solving the combined-order design (COMD) problem are developed. The obtained results will be presented in order to illustrate the application and the feasibility of the suggested approach.

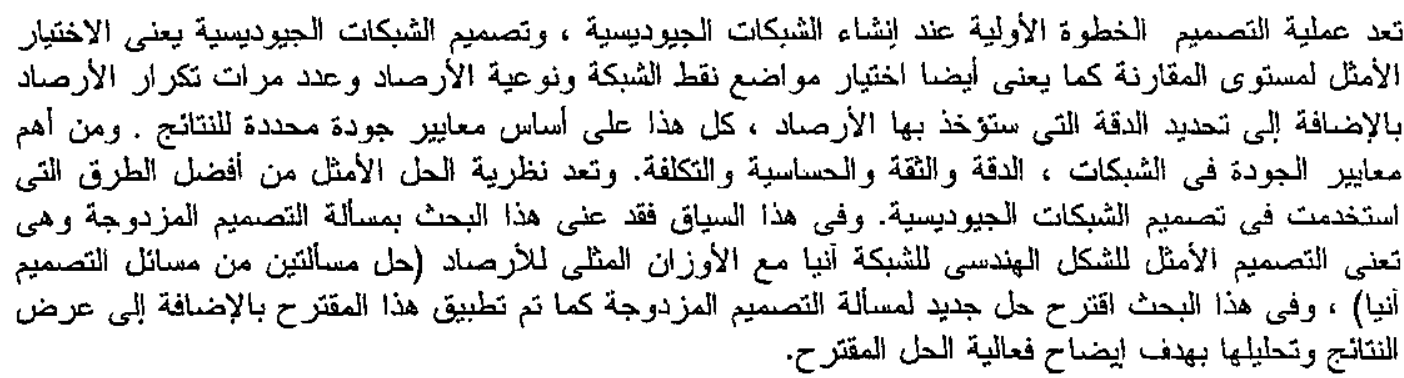

Keywords: Optimization - combined order design (COMD) problem - Criterion matrix - semianalytical approach.

\section{INTRODUCTION}

In the Combined Design (COMD) Problem, the FirstOrder Design Problem and the Second-Order Design Problem have to be optimally solved simultaneously .The main objective of the current study is the solution of the combined order design (COMD) Problem, which can be classified, according to the present study, into three types namely; trial and error approach, analytical approach and semi-analytical approach. A detailed discussion about the combinedorder design (COMD) problem and the steps followed for its solution are given.
In this paper, a new proposed approach for solving the combined order design problem has been suggested. Also the solution of the combined-order design problem has been performed using a suggested semi-analytical approach.

\section{THE COMBINED-ORDER DESIGN} PROBLEM:

In the Combined - Order Design (COMD) Problem (Grafarend et al., 1979), both the First-Order Design Problem and the Second-Order Design Problem have to be optimally solved simultaneously. This problem is classified, according to the present study, into three 
types: trial and error approach, analytical approach, and semi analytical approach.

Trial and Error approach: which solves the network under different point positions and different observational plans ((FOD) problem) and obtain the weight matrix ((SOD) problem) for every case until an appropriate network design is accomplished.

Analytical approach: which solves the network with optimal point locations (FOD) problem and obtain the suitable weight matrix (SOD) problem fully analytically through a mathematical procedure.

Semi Analytical approach: which solves the firstorder design (FOD) problem using the trial and error technique to get the optimum observational plan and obtain the optimum weight matrix (SOD) problem through fully analytical mathematical procedure.

\subsection{THE TRIAL AND ERROR APPROACH:}

The conventional approach to designing a geodetic control network may be summarized through the following steps (Kuang, 1992):

Step $l$ The first step is to select preliminary locations of control stations on a map or on photographs based on the specific needs of survey project control required by the agency that requests the control.

Step 2. Perform a preliminary field reconnaissance based on the available instrumentation to determine the possible connections between network stations by geodetic observables.

Step 3 The proposed station locations and geodetic observables in steps 1 and 2 constitute an initial design of the network. According to the initial design, hypothetical precision or weights of observables are then used to simulate the quality of the network. Then, improvements to the initial design are introduced, when necessary, by either increasing or decreasing the types (FOD) and numbers (SOD) of geodetic observables initially proposed in step 2 until the simulated network quality meets or exceeds the project criteria set by the client requesting the control.

Step 4. Perform a field reconnaissance to examine the physical possibilities of the simulated network. Control stations are temporarily marked on ground, If conventional terrestrial geodetic observables are proposed, they require intervisibility between network stations. If the GPS technique is to be used, the station site should be wide open, i.e., no existing obstruction should be allowed to block the signals from the GPS satellites. In case a simulated network station is impossible to construct in nature, alternatives are sought and recorded on the map.
Step5 Step (3) and step (4) are iterated until an appropriate network design is accomplished

After step (5), the network is then monumented and surveyed and finally analyzed using the theory of network analysis and then presented to the network user.

\subsection{ANALYTICAL APPROACH:}

In order to develop an analytical approach for network optimal design, the improvements to an initial design must be described by mathematical terms. Let us consider a network in two-dimensional space and assume that approximate locations of network points with approximate coordinates $\left(x_{0}, y_{0}\right)$ were selected from a reconnaissance according to the topography and/or project survey control needs. The configuration of the network can be optimized by introducing improvements $\left(\Delta x_{i}, \Delta y_{i}\right)$ in the coordinates of each point. As for the solution of weights of observations, we can also start with a set of approximate weights $p_{0}$ that can be realized with least efforts, then introduce improvements $\left(\Delta p_{j}\right)$ in order to achieve the design criteria. The final optimal positions and weights are then obtained by adding the solved for optimal improvements to their corresponding initial values as follows:

$$
\left.\begin{array}{l}
x_{i}=x_{i}^{o}+\Delta x_{i} \\
y_{i}=y_{i}^{o}+\Delta y_{i}
\end{array}\right\} \quad, i=1,2, \ldots, m
$$

and

$$
p_{i}=p_{i}^{\prime \prime}+\Delta p_{i} \quad, \quad i=1,2, \ldots, n
$$

\subsection{SEMI-ANALYTICAL APPROACH:}

In the semi-analytical approach, the observational plan (FOD) Problem is solved using trial and error technique and the optimum weight matrix (SOD) Problem is solved fully analytically through a mathematical procedure. . For solving the observational plan (FOD) problem, the trial and error technique can be applied using one of two methods. In the first method, we study the influence of each single observation on the quality of the network. In the second method, we start with all possible observations, after that, reduce the observation plan to the minimum observations and get the real variance-covariance matrix for the two designs, then, compare the different designs with each other. In the current study, the second method will be used to solve the COMD problem.

\section{SOLVING THE COMBINED-ORDER} DESIGN PROBLEM USING THE SEMIANALYTICAL APPROACH:

The solution of the Combined-Order Design Problem is classified into three types: Trial and Error 
approach, Analytical approach and Semi-analytical approach. In the current study, we perform a combined first-order and second-order design using a semi-analytical technique. In this case, the observational plan and the weight vector have to be optimally determined in order to achieve the required precision criteria. The optimization algorithm starts with an initial design that proposes the approximate locations of network stations as well as the types and precision of observations to be made. As for the observing plan, we propose for the purpose of generality, to measure all the possible spatial distances and directions (all possible observations). The initial precisions of the proposed observations may be given arbitrarily. Then, the observation plan is reduced to the minimum possible observation plan. After that, the optimization process is executed using a criterion matrix to solve for the optimal weight to be introduced to the initially given approximate coordinates and to all the initially given weights of observables. Finally the error ellipses are computed in each case separately.

In the present study, we propose to use a COMD optimization strategy in five steps as the following:

Step 1 The first step is starting with an observation plan, which includes all possible observations (directions and distances), and getting the real variance-covariance matrix $\mathrm{Qx}$.

Step 2. Reduction of the proposed observation plan (directions and distances) to the minimum observation plan (directions only or distances only).

Step 3 Choosing a criterion matrix for a network to get the optimum weight of a geodetic network. A criterion matrix can be structured by different ways, for instance, the Taylor-Karman structure, the chaotic structure and the modification of the present variance-covariance matrix. In the present study, the Taylor-Karman structure will be used for getting the required criterion matrix.

Step 4 Solving the SOD Problem of the two different designs (the all-possible observations and the reduced observations). The SOD Problem can be solved by two basic approaches: The leastsquares solution and the mathematical programming techniques.

Step 5 Comparing the results of the two different designs to select the best observational plan and the best weight matrix that agree with the user requirements.

\section{THE SOLUTIUON STEPS OF THE COMBINED-ORDER DESIGN PROBLEM USING THE SEMI-ANALYTICAL APPROACH:}

In the current study, the Combined-Order Design problem will be solved using the semi-analytical approach, through the following steps:

1) Computation of the design matrix $\left(A_{1}\right)$ for the all possible observations:

This is achieved by using the given approximate values of parameters to evaluate the partial derivatives within the design matrix $\left(A_{1}\right)$.

2) Calculation of the initial weight matrix for all possible observations

The initial weight matrix $\left(P_{1}\right)$ for single observation (assuming the a priori variance factor $\sigma_{0}^{2}=1.0$ ) can be computed from the suggested observational plan.

3) Computation of the real variance-covariance matrix $\left(C_{1}\right)$ :

The variance-covariance matrix of the estimated coordinates can be expressed (assuming the variance factor $\sigma_{0}^{2}=1.0$ )by:

$C_{1}=\left(A_{1}^{\mathrm{T}} P_{1} A_{1}+D D^{T}\right)^{-1}-G\left(G^{T} D D^{T} G\right)^{-1} G^{T}$

where

$\mathrm{G}$ : is the inner constrained network datum matrix

$D$ : is the minimum constrained network datum matrix

$P_{1}$ : is the initial weight matrix

Note that: for the free network case, the datum matrix (D) $=0.0$, the variance-covariance matrix can be computed by:

$C_{1}=\left(A_{1}^{T} P_{1} A_{1}\right)^{+}$

4) Specifying the criterion matrix $\left(Q_{1}\right)$ :

The criterion matrix can be performed using one of the objective functions that detailed in Rahil et.al (2004). It is to be mentioned here, that the criterion matrix has been computed in the current study using the Taylor-Karman structure.

5) Determination of the optimal weight matrix $\left(\mathbf{P}_{\mathrm{F} 1}\right)$ :

The optimal weight vector $\left(\mathbf{P}_{\mathrm{F} 1}\right)$ can be determined by solving the SOD Problem.

6) Computation of the repetition numbers for the observations:

From the resulting weights vector $\left(\mathrm{P}_{\mathrm{FI}}\right)$, the required variances for the observations can be obtained by: 


$$
\left(\sigma_{\imath}^{2}\right)_{\text {req. }}=\frac{\sigma_{0}^{2}}{P_{F 1}}
$$

Then, the repetition number of any observation is obtained by:

$$
n_{i}=\frac{\left(\sigma_{s}^{2}\right)_{i}}{\left(\sigma_{i}^{2}\right)_{\text {req. }}}
$$

where:

$\left(\sigma_{i}^{2}\right)_{\text {req. }}:$ is the required variance of the observation.

$\left(\sigma_{\mathrm{S}}^{2}\right)_{\mathrm{i}}:$ is the variance of the single observation.

\section{7) Computation and plotting of the final criteria:}

After calculation of the real variance-covariance matrix, the actual criteria can be computed. Then, the criteria ate plotted (e.g., the error ellipses) to illustrate the results.

8) Reduction of the observational plan to the minimum (available) observations:

The design of the observational plan is changed to the minimum (available) observations. In our case here, the hybrid network will be reduced to a trilateration network.

\section{9) Reiteration of steps:}

The steps 1 through 7 are repeated for the reduced observations, to obtain the following:

Design matrix $\left(A_{2}\right)$, initial weight matrix $\left(\mathrm{P}_{2}\right)$, the present variance-covariance matrix, the optimal weight matrix $\left(P_{F 2}\right)$, the repetition numbers $\left(n_{i 2}\right)$ and finally, computation and plotting of the final criteria.

10) Compare the results of the two different designs:

Compare the computed quality measures of the two designs (the all-possible observations and the reduced observations), with the required specifications of the network, to select the best design that agrees with the specifications of the project with minimum cost.

Figure 1 shows a Diagram for the solution steps of the case study, which will be solved using the COMD Problem.

\section{DESCRIPTION OF THE CASE - STUDY}

Figure 2 shows a braced quadrilateral figure. The approximate coordinates of netpoints are listed in Table 1. It is assumed that an EDM instrument would be used to measure all the distances with nominal accuracy from:
$\sigma_{\mathrm{S}}=3.0 \mathrm{~mm}+2.0 \mathrm{ppm} . \mathrm{S}(\mathrm{mm})$, where $\mathrm{S}$ is the distance computed from the approximate coordinates and a theodolite with a precision equal 1.0 second for any observed angle. All the semi-major axes of the absolute errors of the points are required to be 1.00 $\mathrm{mm}$.

Table 1 The approximate coordinates of the netpoints

\begin{tabular}{|l|l|l|}
\hline Point & $\mathrm{X}(\mathrm{m})$ & $\mathrm{Y}(\mathrm{m})$ \\
\hline 1 & 1200.00 & 1300.00 \\
\hline 2 & 1400.00 & 2100.00 \\
\hline 3 & 2200.00 & 2200.00 \\
\hline 4 & 2100.00 & 1100.00 \\
\hline
\end{tabular}

\section{RESULTS AND DISCUSSIONS}

Remember that, the COMD problem is to solve for both the network configuration (FOD problem observation plan) and observations accuracy (SOD problem - weights), in order to meet the user requirements.

Here, in our case, the observational plan is studied in different cases of designs, starting with the conventional design of hybrid network (distances and directions), after that, the design of the observational plan will be changed to a minimum observations (trilateration) and get another new output each time. A comparison of the two different designs will be verified with some selected measures of global precision, like for instance, the variance covariance of the coordinates of all network points and the absolute error ellipses of all network points.

The case study will be solved for the free network concept with the non-linear programming method. The precision criteria formed, as a unit matrix will be considered (T.K structure for the free networks). Figure 3 shows the absolute error ellipses of the COMD problem with the all-possible observations, while Figure 4 shows the absolute error ellipses of the COMD problem after the reduction of the observation plan.

Table 2 and Table 4 give the results (the criteria and the repetition numbers) of the COMD problem with the all possible observations, while Tables 3 and 5 give the results of the COMD problem (the criteria and the repetition numbers) after the reduction of the observation plan. 
Rahil, Abo El Hassan, "A New Proposed Approach For Solving The Combined Order Design Problem"

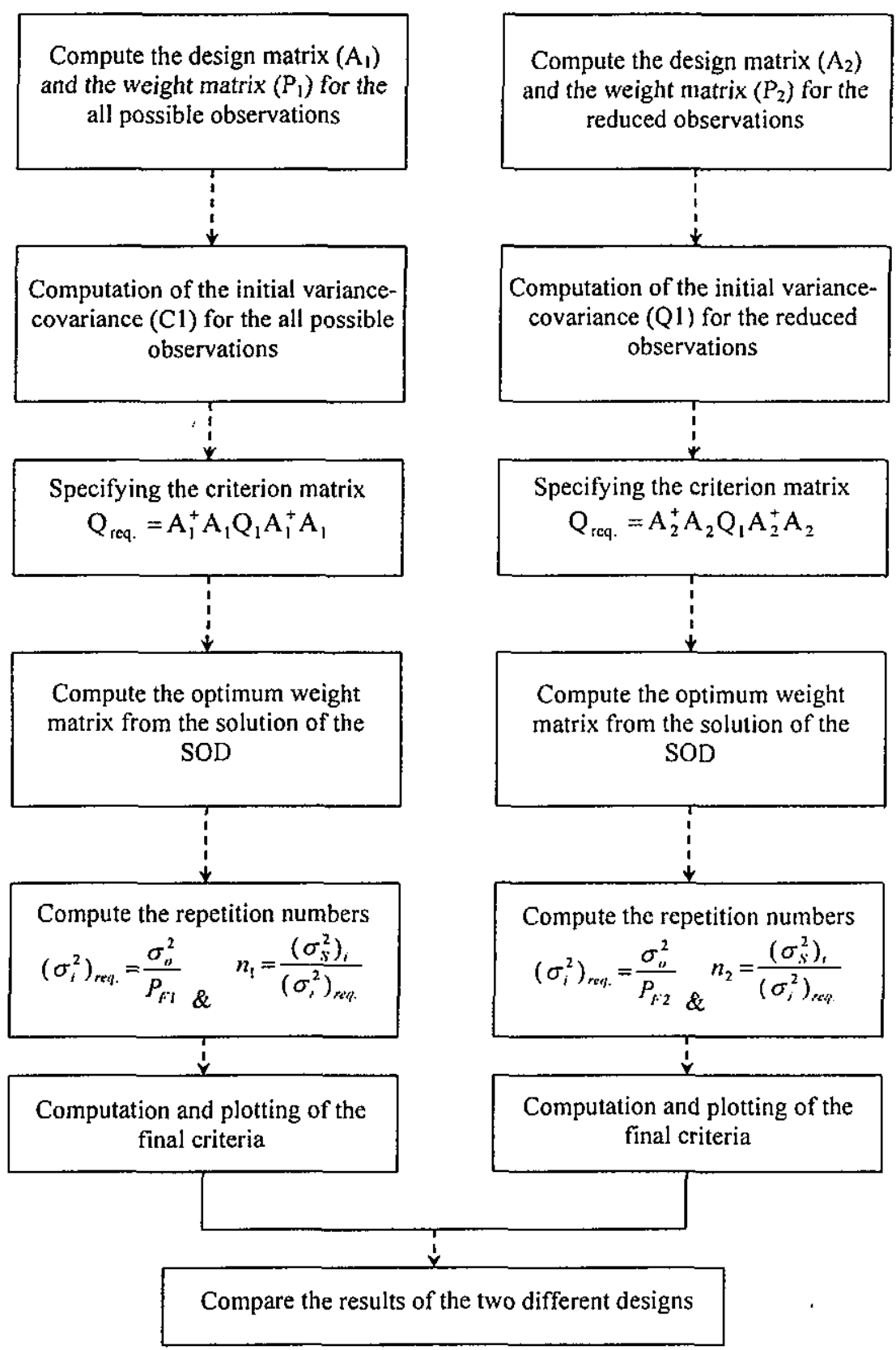

Fig. 1 Diagram for the solution steps of the combined-order design (COMD) problem using the semi-analytical approach.

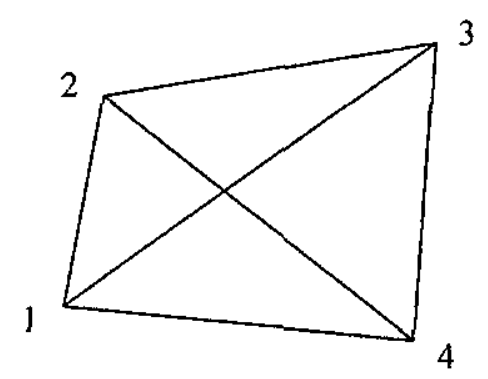

It is to be mentioned that the repetition numbers in table 4 and 5 satisfy the required criteria. The variance covariance matrix of the estimated coordinates is obtained from the COMD problem with the all possible observations, that ensures the given criterion matrix was computed as follows:

Fig. 2 Braced Quadrilateral (Horizontal) Figure 
$\sigma_{3}=10^{4 n *}$

$\left(\begin{array}{llll}0.8007 & 0.2269 & 0.0240-0.1079-0.3068-0.2454-0.5179 & 0.1264\end{array}\right)$ $0.22690 .6137-0.0605-0.4510-0.2246-0.2550 \quad 0.0581 \quad 0.0923$ $0.0240-0.06050 .6483-0.1102-0.4775-0.0372-0.19480 .2078$ $\begin{array}{llllll}-0.1079-0.4510 & 0.1102 & 0.7124 & 0.0437 & 0.0906 & 0.1744-0.3519\end{array}$ $-0.3068-0.2246-0.4775 \quad 0.04370 .6727 \quad 0.29490 .1117-0.1140$ $-0.2454-0.2550-0.03720 .09060 .29490 .7209-0.0123-0.5565$ $\begin{array}{lllllllllllll}-0.5179 & 0.0581 & -0.1948 & 0.1744 & 0.1117 & -0.0123 & 0.6011 & -0.2203\end{array}$ $\left.\begin{array}{lllllll}0.1264 & 0.0923 & 0.2078 & -0.3519-0.1140 & -0.5565 & -0.2203 & 0.8161\end{array}\right)$

: In which

Trace $\left(\operatorname{Tr}_{1}\right)=5.5859 * 10^{-6}$

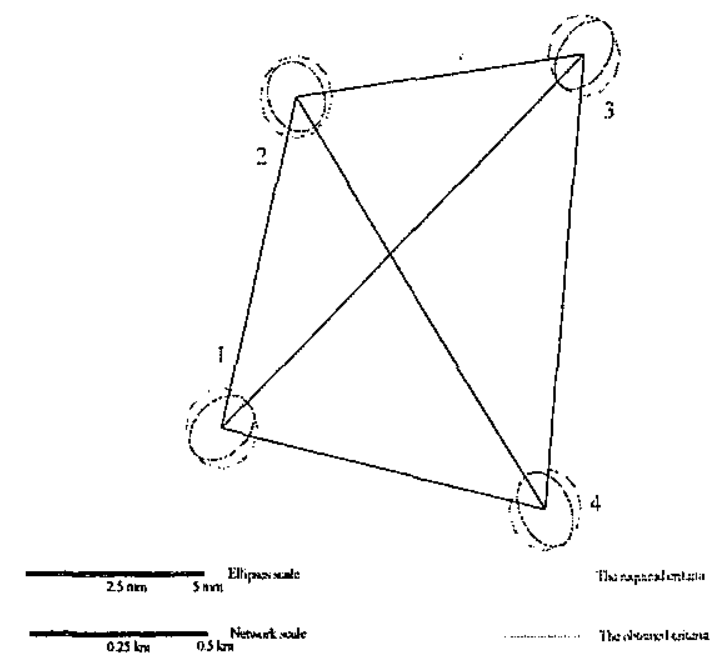

Fig. 3 The results from The COMD With All Possible Observation Plan

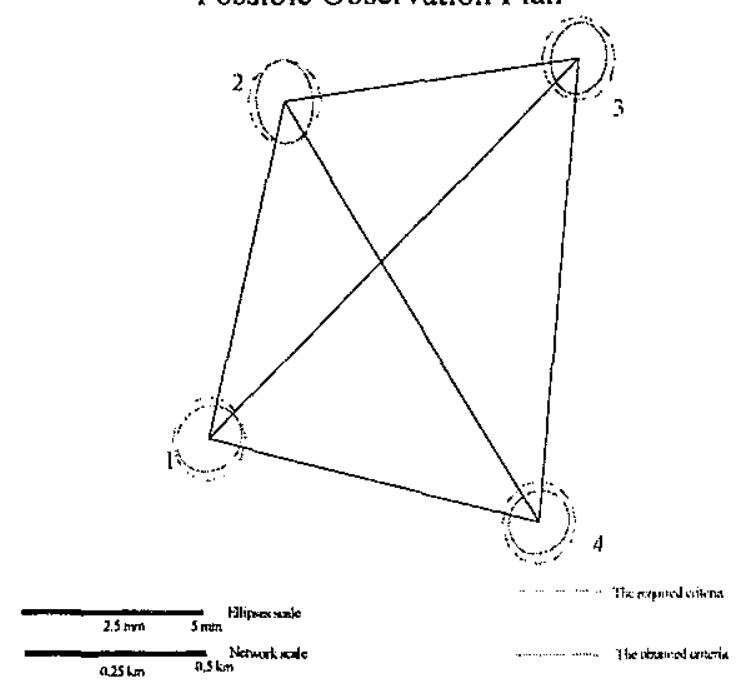

Fig. 4 The results from The COMD With The Reduced Observation Plan

The variance covariance matrix of the estimated coordinates is obtained from the COMD problem after the reduction of the observation plan that ensures the given criterion matrix was computed also :as follows: $\sigma_{2}=10^{\text {h }} *$

$\left(\begin{array}{lll}0.77640 .0854-0.2961 & 0.2493-0.1099-0.1037-0.3703-0.230\end{array}\right)$

$0.08540 .6460-0.1549-0.3532-0.1844-0.06520 .2539-0.2276$

$\begin{array}{lllllll}-0.2961-0.1549 & 0.7625-0.1865-0.3651 & 0.3172-0.1013 & 0.0242\end{array}$

$0.2493-0.3532-0.1865 \quad 0.7864-0.1163-0.3198 \quad 0.0535-0.1134$

$\begin{array}{llllll}-0.1099-0.1844-0.365 !-0.1163 & 0.5816 & 0.0530 & -0.1066 & 0.2477\end{array}$

$\begin{array}{lllllll}-0.1037-0.0652 & 0.3172 & -0.3198 & 0.0530 & 0.7217 & -0.2665 & -0.3366\end{array}$

$\begin{array}{llllll}-0.3703 & 0.2539 & -0.1013 & 0.0535-0.1066-0.2665 & 0.5782 & -0.0410\end{array}$

$\left(\begin{array}{llllll}-0.2310-0.2276 & 0.0242-0.1134 & 0.2477-0.3366 & -0.0410 & 0.6776\end{array}\right)$

In which

Trace $\left(\mathrm{Tr}_{2}\right)=5.5303 * 10^{-6}$

From these results, it can be found that:

1- Both the COMD problem with the all possible observations and with the reduced observation plan yield almost the same results of precision $\left(\operatorname{Tr}_{1}=\right.$ 5.5859* $10^{-6}$ and $\mathrm{Tr}_{2}=5.5303 * 10^{\circ}$ ), that must agree with the specifications of the network, then the more economic one is considered as the optimal design of the observational plan and the weight problem. Suppose that, the two different designs are observed using the same total station instrument under the same field observing conditions, and observing one angle on six arcs needs 18.0 minutes, while observing one distance needs 6.0 minutes. Then: The time required for observing the first design

$=23$ (distances) $\times 6.0+35$ (directions) $\times 18.0$ $=12^{\mathrm{H}} 48^{\mathrm{M}} 00^{\mathrm{S}}$

The time required for observing the second design

$=67$ (distances) $\times 6.0=06^{\mathrm{H}} 42^{\mathrm{M}} 00^{\mathrm{S}}$

From this analysis, it is noticed that the second design (the reduced observation plan) is faster and time saving by about $50.0 \%$ of the time required by the first design (with the all-possible observations). Then, it will be considered as the optimal design of both the observational plan and the weight problem.

2- The semi major axes in case of the all possible observations range from 0.892 at point No. 2 to 1.0 at point No. 3 , while the semi major axes in case of the reduced observation plan range from 0.83 at point No. 4 to 0.99 at point No. 2 .

3- The semi minor axes in case of the all possible observations range from 0.633 at point No. 3 to 0752 at point No. 2, while the semi minor axes in case of the reduced observation plan range from 0.751 at point No. 3 to 0.777 at point No. 1 .

4- The mean area of the absolute error ellipses of the COMD problem with the all possible observations equal $2.07 \mathrm{~mm}^{2}$, while the mean area of the absolute error ellipses of the COMD problem with the reduced observation plan $=2.14 \mathrm{~mm}^{2}$ 
where: The mean area of the absolute error ellipses

$$
=\frac{\sum_{\mathbf{i}=1}^{2 m} \pi \mathbf{a}_{\mathbf{i}} \mathbf{b}_{\mathbf{i}}}{\mathbf{m}}
$$

and: $\quad a_{i}, b_{i}=$ the semi-major and semi-minor axis of the absolute error ellipses.
5- The COMD problem with the all-possible observations has two types of observations (distances and directions), while the COMD problem with the reduced observation plan has one type of observations (distances only). This means that, the process of optimization reduced (for the second design) the types of observations by $50 \%$

Table 2 The criteria of the COMD problem with the all possible observations (distances and directions)

\begin{tabular}{||l|l|l|l|l|l|l|}
\hline \multirow{2}{*}{ Point } & \multicolumn{3}{|c|}{ Required Criteria } & \multicolumn{3}{c|}{$\begin{array}{l}\text { Criteria of the COMD problem with } \\
\text { the all possible observations }\end{array}$} \\
\hline & $\mathrm{a}(\mathrm{mm})$ & $\mathrm{b}(\mathrm{mm})$ & $\Phi$ (Deg.) & $\mathrm{a}(\mathrm{mm})$ & $\mathrm{b}(\mathrm{mm})$ & $\Phi($ Deg. $)$ \\
\hline 1 & 1.0 & 1.0 & 0.0 & 0.976 & 0.680 & 56.20 \\
\hline 2 & 1.0 & 1.0 & 0.0 & 0.892 & 0.752 & 143.11 \\
\hline 3 & 1.0 & 1.0 & 0.0 & 1.000 & 0.633 & 42.66 \\
\hline 4 & 1.0 & 1.0 & 0.0 & 0.977 & 0.681 & 148.02 \\
\hline
\end{tabular}

where: $\mathrm{a}$ : the semi-major axis of the error ellipse. $\quad \mathrm{b}$ : the semi-minor axis of the error ellipse. $\Phi:$ the orientation of the semi-major axis with respect to the y-axis.

\begin{tabular}{|c|c|c|c|c|c|c|}
\hline \multirow[t]{2}{*}{ Point } & \multicolumn{3}{|c|}{ Required Criteria } & \multicolumn{3}{|c|}{$\begin{array}{l}\text { Criteria of the COMD problem } \\
\text { with the reduced observation plan }\end{array}$} \\
\hline & $a(\mathrm{~mm})$ & $\mathrm{b}(\mathrm{mm})$ & $\Phi$ (Deg.) & $a(\mathrm{~mm})$ & $\mathrm{b}(\mathrm{mm})$ & $\Phi$ (Deg.) \\
\hline 1 & 1.0 & 1.0 & 0.0 & 0.91 & 0.777 & 63.68 \\
\hline 2 & 1.0 & 1.0 & 0.0 & 0.99 & 0.767 & 136.84 \\
\hline 3 & 1.0 & 1.0 & 0.0 & 0.86 & 0.751 & 18.56 \\
\hline 4 & 1.0 & 1.0 & 0.0 & 0.83 & 0.751 & 160.24 \\
\hline
\end{tabular}

Table 3 The criteria of the COMD problem with the reduced observation plan (distances only)

Table 4 The repetition numbers from the COMD problem with the all-possible observations (directions and distances)

\begin{tabular}{||l|l||}
\hline \hline Observations & $\begin{array}{l}\text { Repetition no. from the } \\
\text { COMD problem with the } \\
\text { all possible observations }\end{array}$ \\
\hline Distances & 4 \\
\hline $1-2$ & 4 \\
\hline $1-3$ & 4 \\
\hline $1-4$ & 4 \\
\hline $2-3$ & 3 \\
\hline $2-4$ & 4 \\
\hline $3-4$ & \\
\hline Angles & 4 \\
\hline $2-1-3$ & 2 \\
\hline $3-1-4$ & 1 \\
\hline $2-1-4$ & 1 \\
\hline $3-2-4$ & 2 \\
\hline $4-2-1$ & 1 \\
\hline $3-2-1$ & 3 \\
\hline $2-3-1$ & 8 \\
\hline $1-3-4$ & 1 \\
\hline $2-3-4$ & 7 \\
\hline $1-4-2$ & 4 \\
\hline $2-4-3$ & 1 \\
\hline $1-4-3$ & \\
\hline
\end{tabular}

Table 5 The repetition numbers from the COMD problem with the reduced observation plan (distances only)

\begin{tabular}{|l|l|}
\hline Distances & $\begin{array}{l}\text { Repetition no. from the COMD } \\
\text { problem with the reduced } \\
\text { observation plan }\end{array}$ \\
\hline \hline $1-2$ & 9 \\
\hline $1-3$ & 12 \\
\hline $1-4$ & 11 \\
\hline $2-3$ & 10 \\
\hline $2-4$ & 13 \\
\hline $3-4$ & 12 \\
\hline
\end{tabular}

\section{CONCLUSIONS:}

The present mathematical model of the COMD problem which deals with the simultaneous optimization of network configuration (all possible observations) and observational weights, for practical applications, after appropriate network quality criteria are given. This optimization procedure gives the optimal weights or standard deviations for each observation as well as the available observational plan where the positions of the netpoints selected in reconnaissance can not be changed. This is practical, 
especially for engineering network, since the general locations of the network points are fixed.

In this paper, the combined-order design problem has been solved using a semi analytical approach proposed in this paper.

According to the results of this study, the following conclusions can be drawn:

- The obtained results show the importance of the combined order design (COMD) problem to choose the optimal observation plan and determine the optimal weights of observations that will satisfy the pre-set network quality with the minimum cost.

- The present case study shows the feasibility of the suggested semi-analytical approach for the solution of the combined order design (COMD) problem.

- The solution of the case study has proved that, the suggested approach for the solution of the combined order design (COMD) problem is considerably simple and economic. Therefore one can suggest using the adopted semi-analytical approach for the solution of the combined order design (COMD) problem.

\section{REFERENCES}

[1] Allman, J.S. and Hoar, G.J. (1973). "Optimization of geodetic networks". Survey Review XXП, 167.

[2] Amiri Seemkooei, A. (2001). "Comparison of reliability and geometrical strength criteria in geodetic networks." Journal of Geodesy (200I), 75: $227-233$.

[3] Ashkenazi, V. (1974). "Criteria for optimization; a practical assessment of a free network adjustment." Bollietino Di Geodesia E Scienze Affini, No. I.

[4] Banov, B. (1982). "A special method to derive a criterion matrix". Proceeding of S.G.5 B s Meeting, FIG, $7^{\text {th }}-9^{\text {th }}$ July 1982, Aalborg University Center, Danmark.

[5] Blaha, G. (1979). "Discussion of five methods for adjusting free networks." Nova University, Ocean Sciences Center, Dania, Florida, 33004.
[6] Crosilla, F. (1983). "A criterion matrix for the second order design of control networks." Bulletin Geodesique 57: 226-239.

[7] Cross, P.A. and A. Fagir (1982). "Procedures for the first and Second Order Design of vertical control networks." Proc. Survey Control Networks, Heft 7, Munchen.

[8] Doma, M, I (2004) “ Application of optimization theory to geodetic networks using different orders design problems " $\mathrm{M}$. Sc. thesis, Faculty of Engineering, Shebin El-Kom, Minufia University, Egypt.

[9] Ghoneim A., (1999). "Numerical efficient solutions of the second-order design (the optimal weights) of geodetic networks."M.Sc.thesis, Faculty of Engineering, Shebin El-Kom, Minufia University, Egypt.

[10] Grafarend, E. and Schaffrin, B. (1979). "Kriterion-Matrizen I". ZFV 104, 133-149.

[11] Kuang, S.L. (1992). "A new approach to the Optimal Second Order Design of geodetic networks." Survey Review, 31(243), 279-288.

[12] Mousa, A.K. (1992). "Design of geodetic networks for monitoring recent crustal movements using the optimization theory." M.Sc. Thesis, Faculty of Engineering, Ain Shams University, Cairo, Egypt.

[13] Nassar, M.M. (1984). "Matrix treatment of adjustment computations." Dept. of Public Works, Lecture Notes No. 4, Faculty of Engineering, Ain Shams University, Cairo, Egypt.

[14] Nassar, M.M. ; Shaker, A.; and Asal, F. (1994) "Successive solutions for different orders of the optimal design problems for local horizontal geodetic monitoring networks, using simulation process." Proceedings of the $2^{\text {nd }}$ Conference on Civil Engineering, Military Technical College, 8-10 Nov. 1994.

[15] Rahil, A.M., El-Gohary, A. and Ismail, M. (2004). "An alternative approach to obtain a criterion matrix for the solution of the secondorder design problem of geodetic networks" CERM, Vol. 25, No. 6, 2004, El-Azhar University, Faculty of Engineering, Cairo, Egypt 